\title{
El instrumento CODICE-Derecho y su aplicación en la Facultad de Derecho de la Universidad de Chile
}

\section{CODICE-Derecho measuring instrument and its application in the Faculty of Law of Universidad de Chile}

\author{
Marcela Cabrera Pommiez
}

\begin{abstract}
Resumen: se presentan las características y el diseño del instrumento denominado CODICE-Derecho, el cual mide comprensión de lectura y escritura de los estudiantes de primer año de la carrera de Derecho. El énfasis de este instrumento es que está orientado específicamente a estudiantes de Derecho, pues la selección de textos proviene de manuales y revistas especializadas del área, con lo cual se busca responder al principio de vincular las habilidades comunicativas a la disciplina. Además, se presentan los resultados, en las secciones de Lectura y Escritura, de la cohorte 2011 y se interpretan estos resultados a la luz de los niveles de desempeño descritos para cada sección.
\end{abstract}

Palabras clave: comprensión lectora, evaluación, discurso académico.

Abstract: it presents the features and the design of the instrument called CODICE-Derecho, which measures the reading comprehension and the writing of students in the first academic year of Law degree. This instrument is specifically aimed to law students, as the selection of texts comes from specialized textbooks and journals in the area in order to fulfil the requirement of linking communication skills to discipline. In addition, main results in reading comprehension and writing of the 2011 cohort are presented and these results are interpreted in the light of the performance levels described for each section.

Key words: Reading comprehension, evaluation, academic discourse.

\section{Introducción}

La palabra CODICE es un acrónimo que condensa la frase nominal "competencias discursivas de comprensión y escritura", y consiste en un instrumento diseñado por el Departamento de Evaluación, Medición y Registro Educacional de la Universidad de Chile (DEMRE) para medir los niveles de comprensión de lectura y de escritura que poseen los alumnos de primer año de una carrera universitaria, por lo cual tiene un carácter eminentemente diagnóstico y sus aplicaciones se han llevado a cabo durante el primer semestre.

Antes de abordar la situación de los estudiantes cuando ingresan a la universidad es conveniente considerar el aporte de la educación media en este sentido, pues sabemos que

\footnotetext{
${ }^{1}$ Profesora de Castellano y Licenciada en Educación, Pontificia Universidad Católica de Chile; Magister en Lingüística Hispánica, Universidad de Chile; DEA (Diploma de Estudios avanzados) en Sociolingüística, Universidad de Valladolid. Correo electrónico: mecabrera@u.uchile.cl.
} 
prepara a los estudiantes para que desarrollen sus competencias comunicativas, entre otras muchas competencias, como se aprecia en la cita extraída del Marco Curricular vigente:

La propuesta formativa de este sector [Lenguaje] apunta hacia el desarrollo de las competencias comunicativas requeridas por los estudiantes para su desarrollo integral.

Dado que el lenguaje es la base de las demás áreas del saber y constituye en sí mismo una herramienta fundamental para la integración de las personas en una sociedad moderna, participativa y democrática, se propicia el desarrollo del pensamiento crítico y reflexivo, la creatividad y el diálogo. Esto implica enriquecer el lenguaje con el que los estudiantes ingresan al sistema, ampliando y mejorando la comunicación oral y el acceso al lenguaje escrito a través de la lectura y escritura. ${ }^{2}$

Entonces, es el sector de Lenguaje el encargado del fomento de la competencia comunicativa, preparando a los estudiantes para desenvolverse en la sociedad actual como ciudadanos con capacidad de diálogo. Sin embargo, este desarrollo no siempre resulta adecuado para enfrentar las exigencias académicas del primer año de universidad, pues aumenta tanto la cantidad de contenidos por estudiar como la complejidad de los mismos. Entonces sucede que los estudiantes se enfrentan a su realidad como lectores y productores de textos académicos: esta realidad usualmente indica que hay deficiencias, que las pruebas de desarrollo no se abordan correctamente y los textos que deben leer les resultan ajenos y difíciles. Se genera, a partir de estas deficiencias detectadas, la necesidad de revisar los métodos de lectura y estudio, que no es otra cosa que la revisión de las estrategias cognitivas de cada estudiante para enfrentar una exigencia mayor que la que habían conocido en los años de colegio.

Por otra parte, resulta también fundamental que las autoridades universitarias escritura, cuando ingresan a una determinada carrera, pues con una eficiente detección inicial se pueden adoptar políticas adecuadas, que apoyen a los estudiantes en el proceso de incorporarse a la vida académica, la cual tiene características propias como: requerimiento de lectura rápida y eficiente de volúmenes de información mayores; comprensión de textos pertenecientes al discurso académico y expresión adecuada del pensamiento a través de la escritura y la oralidad, con un nivel de formalidad propio de la lengua estándar y distante del diálogo coloquial que es tan familiar a los jóvenes de veinte años.

En este escenario, el instrumento CODICE-Derecho surgió el año 2009 para responder a la necesidad de evaluar las habilidades de lectura y escritura de los estudiantes que ingresan al primer año de la carrera de Derecho en la Facultad de Derecho de la Universidad de Chile. De hecho, el objetivo declarado del instrumento, en su marco teórico, es el siguiente:

[...] el objetivo es contar con información sobre los niveles de desempeño en las áreas de lectura y escritura de los estudiantes universitarios del primer año de esta carrera universitaria. ${ }^{3}$

\footnotetext{
${ }^{2}$ COMITÉ DE LENGUAJE Y COMUNICACIÓN, DEMRE. Marco teórico prueba CODICE-Derecho. Inédito. Santiago de Chile: 2011. P.31.

${ }^{3}$ Ibíd.
} 


\section{Un poco de historia}

El año 2009 se iniciaron las conversaciones entre la Facultad de Derecho y el DEMRE, en torno a la idea de realizar una prueba distinta y complementaria a la PSU, que presentara a los estudiantes de esta facultad textos específicos de las ciencias jurídicas para medir comprensión lectora a través de 50 preguntas cerradas; y luego pidiera la elaboración, por escrito, de respuestas a tres preguntas abiertas (o de desarrollo) que versaran sobre los mismos textos estímulo. La iniciativa se concretó en abril del mismo año, con la aplicación de la primera CODICE-Derecho.

El año 2010 se aplicó la prueba CODICE-Universidad de Chile a todos los alumnos de primer año, de todas las carreras al interior de la universidad, incluida la facultad de Derecho, con lo cual se trabajaron temáticas generales y no específicas para el derecho como disciplina. Los resultados de los alumnos se entregaron a cada facultad.

En 2011 se implementó un nuevo diseño en la CODICE-Derecho, que redujo los ítemes de 50 a 40 y modeló el puntaje de acuerdo a tres competencias, estableciéndose preguntas que podían valer, uno dos o tres puntos. Este mismo diseño se aplicó en 2012. Dado lo anterior, los resultados de 2011 y 2012 son fácilmente comparables.

\section{Estructura del instrumento}

\section{a. Los textos}

Cada prueba presenta cinco fragmentos pertenecientes a textos del área de las ciencias jurídicas, extraídos de la bibliografía de los cursos del primer año o de revistas de derecho. Se trata de fragmentos que tengan un sentido completo, para que permitan formular las 43 preguntas necesarias.

En cuanto a modos discursivos, los fragmentos son expositivos y argumentativos. Su extensión es cercana a mil palabras.

\section{b. Las preguntas}

Se trata de 40 ítemes de selección múltiple, de cinco opciones, donde sólo una es la clave. Cada ítem mide una determinada habilidad cognitiva (comprender, interpretar, etc.).

Tres de los textos van acompañados de una pregunta de desarrollo, la que propone una tarea de escritura en torno a un género específico (por ejemplo: texto argumentativo, definición, caracterización), basándose en temas tratados en el texto estímulo. Por ello, el estudiante debe haber leído comprensivamente el fragmento para elaborar una buena respuesta. 
La corrección se basa en rúbricas, que consideran aspectos tales como planteamiento de ideas, cohesión oracional y normas ortográficas, cada uno de los cuales tiene un puntaje determinado.

La colaboración de académicos de la Facultad de Derecho es fundamental en el proceso de construcción del instrumento, pues la selección de textos se realiza en conjunto $\mathrm{y}$, posteriormente, un grupo de profesores que dicta clases en primer año, revisa el instrumento ensamblado con el fin de validarlo.

\section{Comprensión lectora}

Es un proceso mental complejo que se extiende desde la simple decodificación de enunciados hasta llegar a niveles superiores de interpretación. Es decir, cubre un abanico de competencias que van desde la decodificación hasta la construcción del significado del texto, proceso mucho más profundo y complejo.

En este sentido, se habla de lector experto como aquel que posee estrategias para desarrollar un real proceso de comprensión de lo que está leyendo, estrategias que le permitirán obtener la idea principal y las secundarias, realizar inferencias válidas, construir la coherencia global del texto y conectar el contenido de este con sus conocimientos previos. Todo esto se desarrolla gracias a la metacognición, es decir, gracias a un control de los propios procesos cognitivos activados frente a la resolución de un problema.

\section{Competencias de lectura}

Para la prueba CODICE-Derecho se han establecido tres competencias lectoras:

Competencia 1: consiste en extraer información explícita del texto, es decir, se trata de una competencia que apunta a la comprensión literal de lo que aparece expresado en la superficie textual y se manifiesta en recordar hechos, lugares y/o agentes. También implica ubicar un dato particular o dar cuenta de relaciones explícitas entre elementos textuales.

Competencia 2: consiste en analizar y sintetizar información explícita del texto. Implica un primer grado de abstracción con respecto a la competencia 1, pues abarca la descomposición de la información en elementos constitutivos (analizar) y la capacidad de sintetizar en un enunciado una porción de información, así como de elaborar procesos de comprensión de la coherencia local del texto.

Competencia 3: consiste en interpretar la información del texto, asignando una función discursiva a algún elemento textual, además de realizar inferencias válidas y de evaluar la intención comunicativa del emisor. Es el nivel más alto de comprensión lectora, pues implica establecer funciones y relaciones acertadas para el contenido y la forma del texto, que están subsumidas en este. 


\section{Habilidades cognitivas}

Las competencias antes mencionadas se expresan mediante diez habilidades cognitivas, las que describen una actividad específica en el procesamiento de la información textual, como se detalla a continuación:

1. Comprender: entender información explícita de la superficie textual (datos, lugar de la acción, agentes y sus acciones, etc.).

2. Identificar: reconocer elementos y conceptos de orden textual, lingüístico o comunicativo presentes en el texto del cual procede el ítem.

3. Analizar-sintetizar: descomponer un texto en sus partes y elementos constitutivos y resumir la información resultante.

4. Analizar-interpretar: descomponer un texto en sus partes y elementos constitutivos y asignarles valores deducibles y aplicables a la totalidad, asignando un sentido de lectura posible al texto.

5. Sintetizar localmente: resumir o determinar la idea o las ideas centrales de un pasaje determinado de un texto.

6. Sintetizar globalmente: resumir o determinar la idea o las ideas centrales de un texto.

7. Inferir localmente: concluir, derivar información implícita desde la información explícita contenida en un pasaje determinado del texto estímulo.

8. Inferir globalmente: concluir, derivar información implícita desde la información explícita contenida en la totalidad del texto estímulo.

9. Interpretar: determinar la función o finalidad de una idea, elemento textual o de un aspecto relacionado con el contexto del estímulo, en tanto asignación de un sentido de lectura posible.

10. Evaluar: coemitir o coproducir juicios valorativos con relación a la forma y el contenido aplicables al tex to estímulo.

A su vez, cada habilidad implica ciertas tareas de lectura, como se presentan:

Tabla 1. Tareas de lectura asociadas a cada habilidad

\begin{tabular}{|l|l|rc|}
\hline \multicolumn{2}{|c|}{ Habilidad lectora } & \multicolumn{2}{c|}{ Tarea o actividad } \\
\hline 1. & Comprender & $\checkmark$ & $\begin{array}{l}\text { Entender la información explícita de un texto o un } \\
\text { fragmento expositivo o argumentativo. }\end{array}$ \\
\hline 2. & Identificar & $\checkmark$ & Localizar o detectar información explícita del texto o \\
\hline
\end{tabular}




\begin{tabular}{|l|l|l|}
\hline & & del fragmento expositivo y/o argumentativo. \\
\hline
\end{tabular}

EL INSTRUMENTO CODICE-DERECHO Y SU APLICACIÓN EN LA FACULTAD DE DERECHO DE LA UNIVERSIDAD DE CHILE

Continuación Tabla 1. Tareas de lectura asociadas a cada habilidad

\begin{tabular}{|c|c|c|}
\hline \multicolumn{2}{|r|}{ Habilidad lectora } & Tarea 0 actividad \\
\hline 3. & Analizar-sintetizar & $\begin{array}{l}\checkmark \text { Descomponer un texto expositivo y/o argumentativo } \\
\text { en sus partes y elementos constitutivos con el fin de } \\
\text { obtener una conclusión. }\end{array}$ \\
\hline 4. & Analizar-interpretar & $\begin{array}{l}\checkmark \text { Descomponer un texto expositivo y/o argumentativo } \\
\text { en sus partes y elementos constitutivos con el fin de } \\
\text { asignarles un sentido o valor. }\end{array}$ \\
\hline 5. & Sintetizar localmente & $\begin{array}{l}\checkmark \quad \text { Establecer la idea central de un segmento de un texto } \\
\text { expositivo o argumentativo. } \\
\checkmark \quad \text { Resumir las ideas centrales de un fragmento o de un } \\
\text { texto expositivo o argumentativo. }\end{array}$ \\
\hline 6. & Sintetizar globalmente & $\begin{array}{l}\checkmark \quad \text { Establecer la idea central de un texto expositivo o } \\
\text { argumentativo completo. } \\
\checkmark \quad \text { Resumir las ideas centrales de un texto expositivo o } \\
\text { argumentativo completo. }\end{array}$ \\
\hline 7. & Inferir localmente & $\begin{array}{l}\checkmark \text { Obtener información implícita a partir de lo afirmado } \\
\text { en un párrafo o segmento de un texto expositivo y/o } \\
\text { argumentativo. }\end{array}$ \\
\hline 8. & Inferir globalmente & $\begin{array}{l}\checkmark \text { Obtener información implícita a partir de lo afirmado } \\
\text { en un texto expositivo y/o argumentativo completo. }\end{array}$ \\
\hline 9. & Interpretar & $\begin{array}{l}\checkmark \quad \text { Asignar sentido a la información explícita o implícita } \\
\text { a partir de una parte o del total de los contenidos de } \\
\text { un texto expositivo o argumentativo. } \\
\checkmark \quad \text { Asignar sentido a los recursos discursivos o textuales } \\
\text { a partir de una parte o del total de los contenidos de } \\
\text { un texto expositivo o argumentativo }\end{array}$ \\
\hline 10. & Evaluar & $\begin{array}{l}\checkmark \quad \text { Evaluar la actitud del emisor, a partir de lo expresado, } \\
\text { en un texto expositivo o argumentativo. } \\
\checkmark \text { Producir juicios sobre el modo en que el emisor de un } \\
\text { fragmento o texto expositivo o argumentativo } \\
\text { organiza la información. }\end{array}$ \\
\hline
\end{tabular}




\section{Competencias y habilidades}

La relación entre estos dos términos es inclusiva, pues una competencia se define como un conjunto interconectado de habilidades.

A su vez, las competencias tienen una gradación de menor a mayor complejidad, es decir, la competencia 1, que apunta a reconocer y comprender información explícita, presenta una complejidad menor que la competencias 2 y 3 . Esta última es la que presenta tareas de lectura más difíciles, que requieren especialmente de un lector activo, capaz de establecer relaciones y extraer ideas no explícitas en la superficie textual para reconstruir el significado del texto.

Un reflejo de esta complejidad creciente en la comprensión de lectura es la diferencia en la puntuación que reciben las preguntas, como se muestra a continuación:

Tabla 2. Asignación de puntaje según competencia

\begin{tabular}{|l|l|c|}
\hline \multicolumn{1}{|c|}{ Competencia } & \multicolumn{1}{|c|}{ Habilidades cognitivas } & Puntaje por pregunta \\
\hline Competencia 1 & Comprender, identificar & 1 punto \\
\hline Competencia 2 & $\begin{array}{l}\text { Analizar-sintetizar, analizar- } \\
\text { interpretar, Sintetizar localmente, } \\
\text { sintetizar globalmente }\end{array}$ & 2 puntos \\
\hline Competencia 3 & $\begin{array}{l}\text { Inferir localmente, inferir globalmente, } \\
\text { interpretar, evaluar }\end{array}$ & 3 puntos \\
\hline
\end{tabular}

Según el puntaje obtenido, el estudiante se ubica en uno de los cinco niveles de desempeño, que indican el estado de desarrollo de las competencias de lectura, considerando que el mínimo de logro es el 50\% de las respuestas correctas. Los niveles 0,1 y 2 indican que los estudiantes no han logrado un nivel satisfactorio de comprensión lectora. A continuación se presenta la glosa del nivel 5, que corresponde a un desempeño excelente por parte del estudiante:

"Demuestra un desarrollo elevado o superior de habilidades primarias tales como identificar y extraer información de un texto o segmento de texto, y determinar relaciones entre las estructuras textuales y las ideas contenidas en ellos. Presenta una capacidad superior para resumir las ideas entre segmentos de un texto y el texto completo, y extraer información implícita de su contenido. Además, posee capacidad elevada para producir juicios respecto de la información textual como de su estructura". 
EL INSTRUMENTO CODICE-DERECHO Y SU APLICACIÓN EN LA FACULTAD DE DERECHO DE LA UNIVERSIDAD DE CHILE

\section{Escritura}

Frente a las preguntas cerradas de selección múltiple, las tres preguntas de desarrollo tienen la ventaja de que requieren un proceso más complejo de respuesta por parte del estudiante, el que involucra necesariamente otras estrategias, que van más allá de haber comprendido adecuadamente las cinco opciones para elegir la correcta.

La escritura se presenta así mucho más activa y demandante, pues implica aspectos tales como adopción de un punto de vista para orientar la redacción, organización de ideas y su consiguiente jerarquización, elección de un léxico adecuado y pertinente, búsqueda de ejemplos ilustrativos y uso de conectores y marcadores textuales, entre otros. A esto hay que agregar una proceso previo de comprensión lectora, pues la tarea de escritura se basa en alguno de los cinco textos estímulos presentados en la prueba, y, por lo tanto, es a partir de la información aquí presentada que el estudiante elaborará su respuesta, por lo que se asume que no recurre exclusivamente a su enciclopedia mental sino que escribirá un texto mixto, en el cual considere elementos textuales dados y conocimientos propios, con lo cual se espera que sea capaz de obtener inferencias, interpretaciones y evaluaciones de la lectura y las integre en su respuesta.

La ejecución de la respuesta abierta contempla una etapa más o menos breve de planificación; otra de escritura, seguida de la revisión. Se presentan así las etapas propias de la redacción en un ámbito académico, las que se desarrollan con un límite de tiempo (dos horas y media en total) que no permite desarrollar una planificación más extensa y una etapa de reescritura. De acuerdo con el propósito comunicativo medido, existen dos modelos posibles de instrucciones de escritura. Uno de ellos tiene un propósito argumentativo; el otro, expositivo. En el caso de la aplicación 2012, las tres preguntas abiertas son argumentativas por tres razones: primero, la temática de los textos se prestaba para formular preguntas argumentativas; segundo, se consideró que, para desarrollar las capacidades escriturales de los alumnos, la argumentación es un tipo de discurso especialmente adecuado, dada la complejidad de su elaboración; y, tercero, se trata de un discurso propio del quehacer académico del alumnado de la carrera de derecho.

La corrección de estas preguntas se realiza en base a rúbricas, que contemplan aspectos como estructuración de ideas en párrafos, planteamiento de ideas y ortografía. Cada indicador tiene un determinado puntaje, que arroja un total de 25 puntos como máximo por pregunta.

Del mismo modo que en la sección Lectura, en Escritura se han establecido niveles de desempeño de acuerdo con el puntaje obtenido por el estudiante. El nivel 5, denominado Muy adecuado describe un excelente desempeño por parte del estudiante.

El hecho de haber alcanzado puntajes que caben dentro del rango adecuado y muy adecuado en las respuestas dadas a los tres ítemes indica que el estudiante no omitió ninguna de las instrucciones. Junto con ello, sus competencias discursivas, de comprensión de lectura y de manejo discursivo escrito son óptimas, pertinentes y adecuadas. Comprende a cabalidad las instrucciones de los ítemes, desarrolla textos coherentes y cohesionados, formalmente estructurados de manera óptima, los que vincula con atingencia a los estímulos, demostrando una adecuada capacidad de comprensión lectora. Podría aun 
presentar algunos fenómenos aislados y de escasa importancia que influyen en la excelencia de su producción discursiva, aunque esto no contribuye a considerar sus capacidades como insuficientes.

\section{Resultados de la aplicación 2011}

Resultados generales de la aplicación del instrumento

- 390 inscritos, rinden 242 , lo que arroja una asistencia del $62 \%$

- En cuanto a género, los que rinden se desglosan así: 59,9\% hombres y 40,1\% mujeres.

- En cuanto a dependencia educacional, los alumnos de colegios particulares pagados son el 41,7\%; los de colegios particulares subvencionados el 33,5\%; los de colegios municipales el $23,1 \%$. Hay un $1,7 \%$ de estudiantes de los que no se tiene información, pues son de ingreso especial.

Resultados de la sección Lectura

1. Datos generales del grupo

Tabla 3. Resultados generales de lectura del grupo que rindió.

\begin{tabular}{|l|l|l|l|}
\hline $\mathrm{N}$ & 242 & Máximo & 76 ptos. \\
\cline { 1 - 3 } Promedio & 53,41 ptos. & Mínimo & 15 ptos. \\
\cline { 1 - 2 } Desv. Estándar & 11,14 ptos. & \multicolumn{2}{|l}{} \\
\cline { 1 - 3 } & &
\end{tabular}

El promedio de los puntajes obtenidos por los estudiantes es 53,41 puntos, lo que indica que la dificultad de la prueba se situó en $65,9 \%$, es decir, resultó de una dificultad mediana-fácil. El máximo real fue de 76 puntos, mientras el ideal era de 90 puntos.

Aquí es necesario aclarar que al analizar los resultados, se decidió eliminar seis preguntas, debido a que presentaron un indicador estadístico deficiente; esto es, un índice de discriminación (rb) inferior a 0,200. Hecho esto, los totales se modificaron del siguiente modo: la cantidad de preguntas se redujo a 34 y el puntaje máximo a 81 puntos. 


\section{Niveles de desempeño en lectura}

Tabla 4. Resultados en lectura desglosados por nivel de desempeño

\begin{tabular}{|c|c|c|c|c|}
\hline Nivel & Puntaje & Nivel de desempeño & Frecuencia & Porcentaje \\
\hline $\mathbf{0}$ & $0-12$ & Sin evidencia & 0 & $0,00 \%$ \\
\hline $\mathbf{1}$ & $13-26$ & Insuficiente & 6 & $2,48 \%$ \\
\hline $\mathbf{2}$ & $27-40$ & Mínimo & 20 & $8,26 \%$ \\
\hline $\mathbf{3}$ & $41-54$ & Suficiente & 86 & $35,54 \%$ \\
\hline $\mathbf{4}$ & $55-68$ & Bueno & 119 & $49,17 \%$ \\
\hline $\mathbf{5}$ & $69-81$ & Excelente & 11 & $4,55 \%$ \\
\hline TOTAL & & & $\mathbf{2 4 2}$ & $\mathbf{1 0 0 \%}$ \\
\hline
\end{tabular}

Gráfico 1

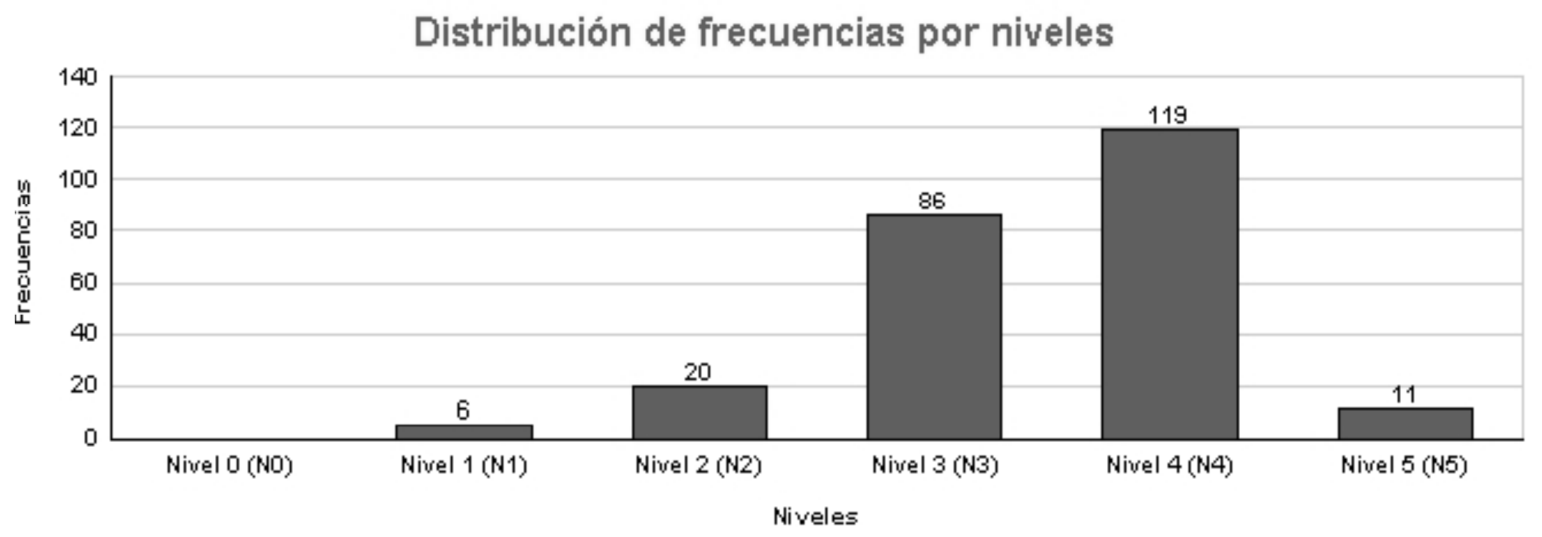

El gráfico 1 muestra los niveles de desempeño de los estudiantes en la sección Lectura. El nivel 3, denominado Suficiente, es el necesario para considerar que el estudiante alcanzó un nivel de logro adecuado en comprensión de lectura, como se aprecia en la glosa correspondiente a este nivel: "Demuestra un desarrollo suficiente de habilidades primarias, tales como identificar y extraer información de un texto o segmento de texto, y determinar relaciones entre las estructuras textuales y las ideas contenidas en ellos. Presenta capacidad suficiente para resumir y correlacionar las ideas entre segmentos de un texto y el texto completo, y extraer información implícita de su contenido. Además, evidencia una capacidad suficiente para producir juicios respecto de la información textual, así como de su estructura". 4

\footnotetext{
${ }^{4}$ COMITÉ DE LENGUAJE Y COMUNICACIÓN, DEMRE. Marco teórico prueba CODICE-Derecho. Inédito. Santiago de Chile: 2011. P. 36.
} 
En este nivel se ubica el 35,54\% de los estudiantes de primer año. El grupo mayoritario está en el nivel Bueno $(49,17 \%$ ) y sólo un $4,55 \%$ se ubica en el nivel superior (nivel 5 o Excelente). Lo anterior demuestra que el nivel de comprensión de lectura de los alumnos de primer año de la Facultad de Derecho tienen, en general, un desempeño notable, agrupado en los niveles superiores, pues sólo un 10,74\% está bajo el nivel de logro establecido, es decir, su comprensión de lectura es deficiente y requieren, por tanto, de estrategias remediales para mejorarla, en vistas a las exigencias académicas que deberán enfrentar.

Resultados por competencias

Tabla 5. Resultados en lectura desglosados por competencias

\begin{tabular}{|c|c|c|c|c|c|c|}
\cline { 2 - 7 } \multicolumn{1}{c|}{} & \multicolumn{2}{c|}{ Competencia 1 } & \multicolumn{2}{c|}{ Competencia 2 } & \multicolumn{2}{c|}{ Competencia 3 } \\
\hline Nivel & Frecuencia & $\%$ & Frecuencia & $\%$ & Frecuencia & $\%$ \\
\hline $\mathbf{0}$ & 2 & 0,83 & 0 & 0 & 0 & 0 \\
\hline $\mathbf{1}$ & 18 & 7,44 & 3 & 1,24 & 10 & 4,13 \\
\hline $\mathbf{2}$ & 41 & 16,94 & 31 & 12,81 & 16 & 6,61 \\
\hline $\mathbf{3}$ & 67 & 27,69 & 84 & 34,71 & 105 & 43,39 \\
\hline $\mathbf{4}$ & 78 & 32,23 & 106 & 43,80 & 101 & 41,74 \\
\hline $\mathbf{5}$ & 36 & 14,88 & 18 & 7,44 & 10 & 4,13 \\
\hline Total & $\mathbf{2 4 2}$ & $\mathbf{1 0 0}$ & $\mathbf{2 4 2}$ & $\mathbf{1 0 0}$ & $\mathbf{2 4 2}$ & $\mathbf{1 0 0}$ \\
\hline
\end{tabular}

Gráfico 2

Distribución de frecuencias de grupo total por niveles y competencias

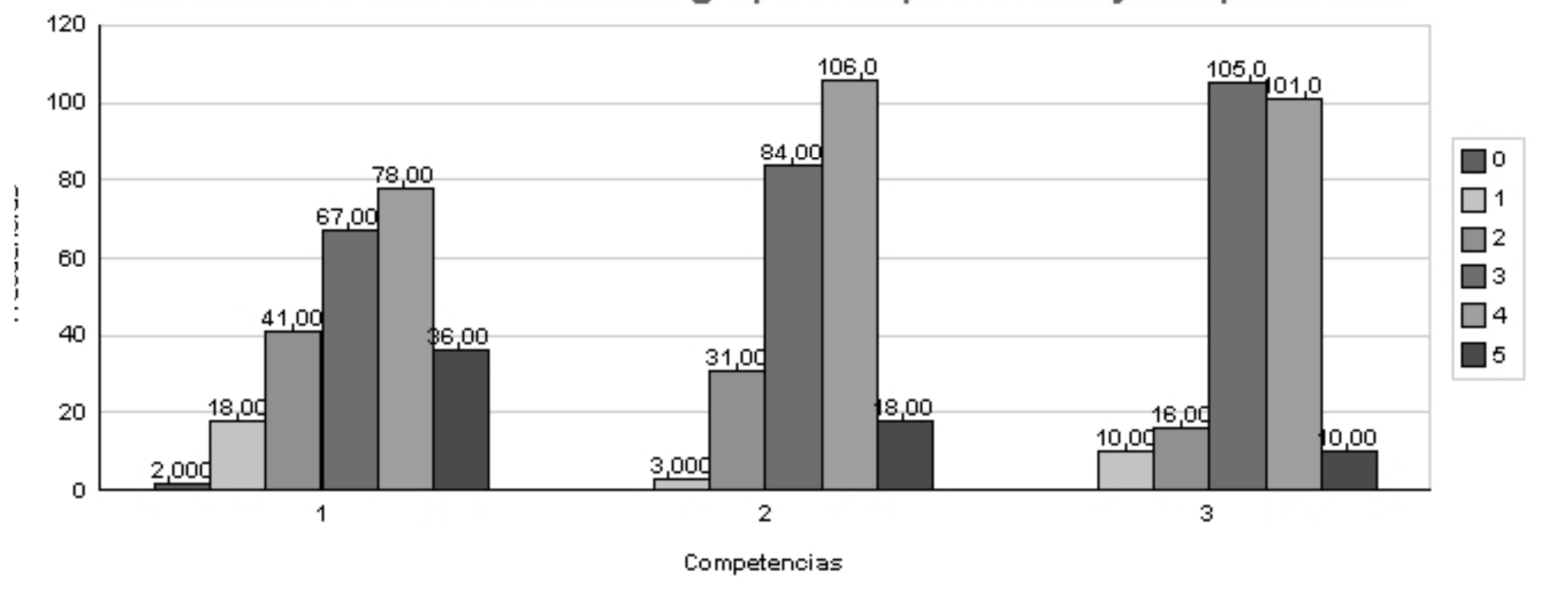


El desglose de distribución de alumnos/as por competencias de comprensión lectora informa que estos se ubican en un nivel alto, especialmente en las competencias 1 y 2 , pues los porcentajes más elevados están en el nivel de desempeño 4 (nivel Bueno), el nivel 5 (Excelente) tiene porcentajes de un $14,88 \%$ para la competencia 1 y de $7,44 \%$ para la competencia 2. La competencia 3, aquella que presenta un nivel de exigencia alto, puesto que implica un buen manejo de la información textual para alcanzar niveles superiores de inferencia, interpretación y evaluación presenta altas cifras en los niveles 3 y 4 (43,39\% y $41,74 \%$, respectivamente).

Resultados por habilidades cognitivas

Tabla 6. Resultados en lectura desglosados por habilidades

\begin{tabular}{|c|}
\hline Niveles de logro por habilidad \\
Nivel 0: insuficiente \\
Nivel 1: suficiente \\
Nivel 2: elevado \\
\hline
\end{tabular}

Habilidades cognitivas

\begin{tabular}{|l|l}
\hline Habilidad 1: Comprender & Habilidad 6: Sintetizar globalmente \\
Habilidad 2: Identificar & Habilidad 7: Inferir localmente \\
Habilidad 3: Analizar-sintetizar & Habilidad 8: Inferir globalmente \\
Habilidad 4: Analizar-interpretar & Habilidad 9: Interpretar \\
Habilidad 5: Sintetizar localmente & Habilidad 10: Evaluar
\end{tabular}

${ }^{5}$ La habilidad 2 no contó con preguntas en esta versión del instrumento. 


\begin{tabular}{|c|c|c|c|c|c|c|c|c|c|c|}
\hline \multicolumn{7}{|c|}{ Resultados por Habilidades } \\
\hline Nivel & \multicolumn{2}{|c|}{$\mathbf{1}$} & \multicolumn{2}{|c|}{$\mathbf{3}$} & \multicolumn{2}{|c|}{$\mathbf{4}$} & \multicolumn{2}{|c|}{$\mathbf{5}$} & \multicolumn{2}{|c|}{} \\
\hline 0 & Frec & $\%$ & Frec & $\%$ & Frec & $\%$ & Frec & $\%$ & Frec & $\%$ \\
\hline 1 & 20 & 8,26 & 8 & 3,31 & 16 & 6,61 & 38 & 15,7 & 154 & 63,64 \\
\hline 2 & 108 & 44,63 & 0 & 0 & 180 & 74,38 & 80 & 33,06 & 71 & 29,34 \\
\hline 3 & 114 & 47,11 & 234 & 96,69 & 46 & 19,01 & 124 & 51,24 & 17 & 7,02 \\
\hline Total & $\mathbf{2 4 2}$ & $\mathbf{1 0 0}$ & $\mathbf{2 4 2}$ & $\mathbf{1 0 0}$ & $\mathbf{2 4 2}$ & $\mathbf{1 0 0}$ & $\mathbf{2 4 2}$ & $\mathbf{1 0 0}$ & $\mathbf{2 4 2}$ & $\mathbf{1 0 0}$ \\
\hline
\end{tabular}

\begin{tabular}{|c|c|c|c|c|c|c|c|c|}
\hline \multicolumn{7}{|c|}{ Resultados por Habilidades } \\
\hline Nivel & \multicolumn{2}{|c|}{7} & \multicolumn{2}{|c|}{$\mathbf{8}$} & \multicolumn{2}{|c|}{$\mathbf{9}$} & \multicolumn{2}{|c|}{10} \\
\hline 0 & Frec & $\%$ & Frec & $\%$ & Frec & $\%$ & Frec & $\%$ \\
\hline 1 & 101 & 41,74 & 58 & 23,97 & 21 & 8,68 & 16 & 6,61 \\
\hline 2 & 120 & 49,59 & 175 & 72,31 & 126 & 52,07 & 142 & 58,68 \\
\hline 3 & 21 & 8,68 & 9 & 3,72 & 95 & 39,26 & 84 & 34,71 \\
\hline Total & $\mathbf{2 4 2}$ & $\mathbf{1 0 0}$ & $\mathbf{2 4 2}$ & $\mathbf{1 0 0}$ & $\mathbf{2 4 2}$ & $\mathbf{1 0 0}$ & $\mathbf{2 4 2}$ & $\mathbf{1 0 0}$ \\
\hline
\end{tabular}

Gráfico 3

Distribución de frecuencias de grupo total por niveles de desempeño y habilidades de lectura

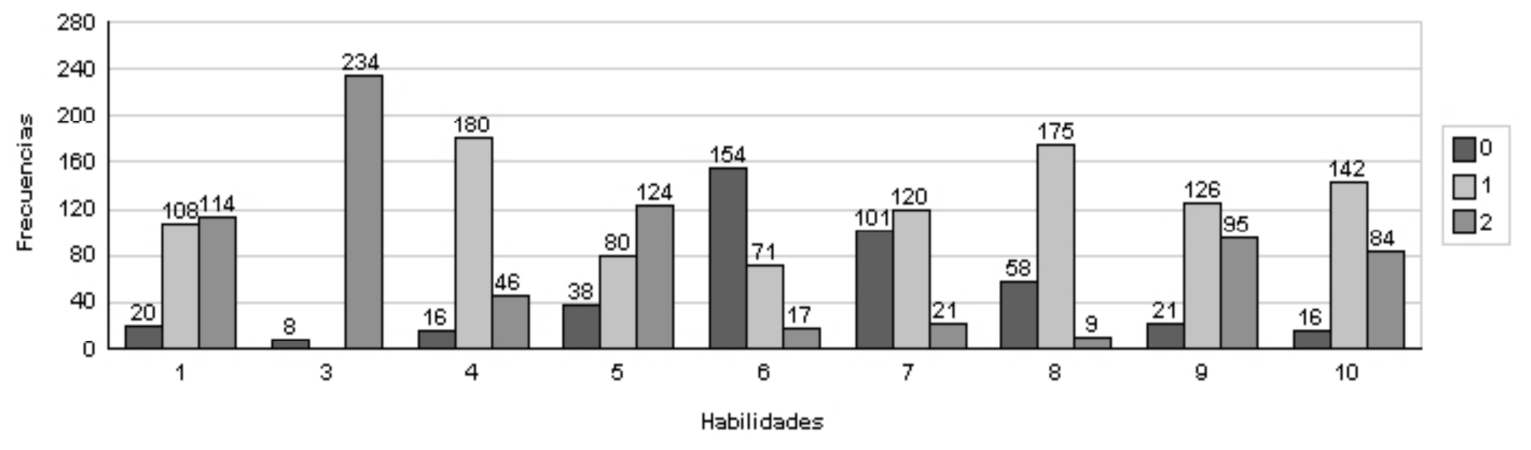


La observación de la tabla 6 y del gráfico 3 permite agrupar las habilidades en cuatro grupos, de acuerdo a su comportamiento:

Grupo 1: excelentes resultados

En este grupo se ubican tres habilidades (comprender, analizar-sintetizar, sintetizar localmente) cuya frecuencia más alta de puntaje se ubicó en el nivel máximo, como se resume a continuación:

\begin{tabular}{|c|c|}
\hline Habilidad & \% de alumnos en nivel 2 \\
\hline Comprender & 47,11 \\
\hline Analizar-sintetizar & 96,69 \\
\hline Sintetizar localmente & 51,24 \\
\hline
\end{tabular}

Grupo 2: buenos resultados

Figuran tres habilidades (analizar-interpretar, interpretar y evaluar) cuyos máximos están en el nivel 1 (Suficiente) y tienen un bajo porcentaje de alumnos en el nivel 0.

\begin{tabular}{|c|c|}
\hline Habilidad & \% de alumnos en nivel 1 \\
\hline Analizar-interpretar & 74,38 \\
\hline Interpretar & 52,07 \\
\hline Evaluar & 58,68 \\
\hline
\end{tabular}

Grupo 3: resultados suficientes

Se trata de los dos tipos de inferencia (local y global), que presentan un buen porcentaje de alumnos en nivel 1, pero la segunda mayoría está en nivel 0 . Hay porcentajes muy reducidos en el nivel 2, que corresponde al mayor dominio (8,68\% para inferir localmente y $3,72 \%$ para inferir globalmente).

\begin{tabular}{|c|c|c|}
\hline Habilidad & \% de alumnos en nivel 1 & \% de alumnos en nivel 0 \\
\hline Inferir localmente & 49,59 & 41,74 \\
\hline Inferir globalmente & 72,31 & 23,97 \\
\hline
\end{tabular}


Grupo 4: resultados deficientes

Se trata de una sola habilidad: sintetizar globalmente. La mayoría se concentra en nivel $0(63,64 \%)$ y la segunda mayoría está en el nivel $1(29,34 \%)$.

Resultados de la sección Escritura

1. Datos generales del grupo

Tabla 7. Resultados generales de escritura del grupo que rindió

\begin{tabular}{|l|l|}
\hline $\mathbf{N}$ & $\mathbf{2 4 2}$ \\
\hline Promedio & 42,27 ptos. \\
\hline Desv. Estándar & 10,81 \\
\hline Máximo & 60 \\
\hline Mínimo & 0 \\
\hline
\end{tabular}

Recordemos que el máximo ideal de la sección Escritura es de 75 puntos. Un estudiante obtuvo el puntaje más alto (60 puntos). El promedio fue de 42,27 puntos, lo que equivale a un $56,36 \%$ de dificultad. Esto indica que la sección presentó una dificultad mediana para los alumnos de primer año.

El total real con que se trabaja en esta sección es 238 estudiantes, pues cuatro de ellos no contestaron ninguna de las tres preguntas abiertas, por lo que no se evalúan y sus hojas se disponen en la categoría "hoja en blanco".

Niveles de desempeño en Escritura

Tabla 8. Resultados en escritura desglosados por nivel de desempeño

\begin{tabular}{|c|c|c|c|c|}
\hline Nivel & Puntaje & Nivel de desempeño & Frecuencia & Porcentaje \\
\hline $\mathbf{0}$ & $5-14$ & Sin evidencia & 2 & 0,84 \\
\hline $\mathbf{1}$ & $15-24$ & Insuficiente & 12 & 5,04 \\
\hline $\mathbf{2}$ & $25-39$ & Mínimo & 50 & 21,01 \\
\hline $\mathbf{3}$ & $40-54$ & Suficiente & 164 & 68,91 \\
\hline $\mathbf{4}$ & $55-66$ & Bueno & 10 & 4,20 \\
\hline $\mathbf{5}$ & $67-75$ & Excelente & 0 & 0 \\
\hline TOTAL & & & $\mathbf{2 4 2}$ & $\mathbf{1 0 0 \%}$ \\
\hline
\end{tabular}


Gráfico 4

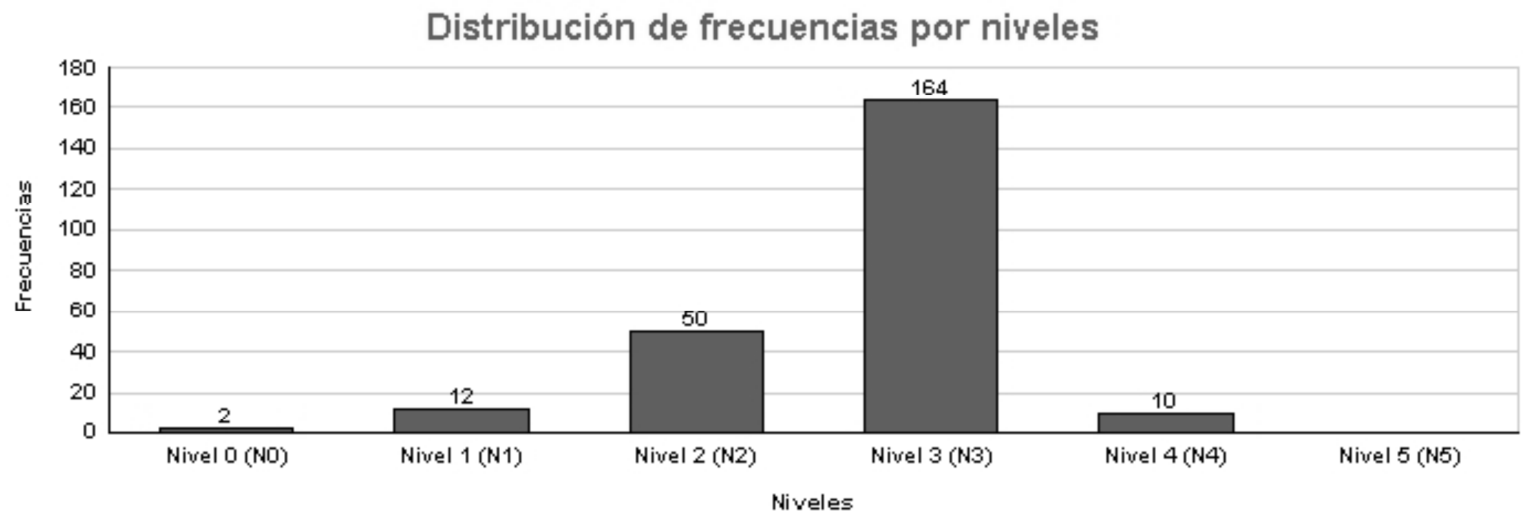

La Tabla 8 muestra que el nivel de desempeño de los alumnos en Escritura es bueno, pues el 73,11\% se ubicó en los niveles de logro adecuados (3 y 4), aunque nadie alcanzó el nivel óptimo (5) y la mayoría se concentra en el nivel Suficiente (nivel 3). Para interpretar correctamente esta información es necesario aclarar que el total de 238 alumnos (y no 242) se explica porque hubo cuatro personas que no respondieron ninguna pregunta de desarrollo, lo cual implica que no se consideran en la distribución de niveles.

Resultados desglosados por pregunta

A continuación se presenta la tabla 9.

Tabla 9. Resultados de preguntas A, B y C

\begin{tabular}{|c|c|c|c|c|c|c|}
\cline { 2 - 7 } \multicolumn{1}{c|}{} & \multicolumn{2}{c|}{ Pregunta A } & \multicolumn{2}{c|}{ Pregunta B } & \multicolumn{2}{c|}{ Pregunta C } \\
\hline Nivel & Frec & $\%$ & Frec & $\%$ & Frec & \% \\
\hline 1 & 0 & 0,0 & 2 & 0,91 & 0 & 0,0 \\
\hline 2 & 30 & 12,71 & 65 & 29,55 & 28 & 14,14 \\
\hline 3 & 177 & 75 & 138 & 62,73 & 129 & 65,15 \\
\hline 4 & 29 & 12,29 & 15 & 6,82 & 41 & 20,71 \\
\hline 5 & 0 & 0,0 & 0 & 0,0 & 0 & 0,0 \\
\hline Total & $\mathbf{2 3 6}$ & $\mathbf{1 0 0}$ & $\mathbf{2 3 6}$ & $\mathbf{1 0 0}$ & $\mathbf{2 3 6}$ & $\mathbf{1 0 0}$ \\
\hline
\end{tabular}

El análisis por ítem revela que la pregunta B resultó más difícil, pues el 30,46\% se ubicó en los niveles 1 y 2 , es decir, no lograron un nivel de escritura adecuado. En cuanto al 
logro, la pregunta $\mathrm{C}$ es la que tiene un mayor porcentaje en el nivel 4 (20,71\%), de lo cual se deduce que fue mejor abordada por los estudiantes.

\section{Conclusiones}

El objetivo principal del instrumento CODICE-Derecho es ofrecer un diagnóstico de las habilidades discursivas de los alumnos de primer año en cuanto a la comprensión de lectura y a la escritura de textos pertenecientes al discurso académico propio del área de las ciencias jurídicas y sociales.

¿Son las habilidades discursivas predictores del éxito o fracaso estudiantil? Esta es una pregunta que interesa a las autoridades universitarias, pues un adecuado manejo de técnicas de comprensión de lectura y de recursos eficaces de escritura podría formar parte de la malla curricular, con el fin de colaborar en la óptima formación de los profesionales del futuro. En este sentido, la prueba CODICE-Derecho es un indicador objetivo del nivel que los alumnos poseen en los inicios de su carrera.

Después de la aplicación de este instrumento, realizada el 2 de abril de 2011 en dependencias de la misma Facultad, vistos y analizados los resultados que se presentan, es posible concluir que:

a) El comportamiento estadístico de los 34 ítemes considerados para el análisis, muestra que el instrumento denominado CODICE-Derecho efectivamente cumple con su rol de establecer un perfil de los estudiantes en cuanto a su desempeño en los ámbitos de lectura y escritura.

b) El desempeño de los estudiantes de primer año en Lectura es muy bueno, pues el $53,72 \%$ se ubica en los niveles más elevados (4 y 5) y el 89,26\% alcanzó el nivel de logro en esta sección, lo que se considera un excelente resultado, pues la población medida demuestra tener adecuados índices de comprensión lectora. Un porcentaje bajo dentro de este 89\%, cercano al 5\%, alcanzó el nivel de excelencia (nivel 5). Estos datos revelan que los alumnos son capaces de comprender la información de la superficie textual, de descomponer esta información y sintetizarla, así como de efectuar procesamientos de mayor complejidad cognitiva (evaluar la intención comunicativa del emisor, interpretar elementos textuales e inferir información no explícita). El análisis por habilidad mostró que la habilidad de sintetizar globalmente es aún débil; en tanto que inferir podría subir su desempeño. Otras habilidades superiores como interpretar y evaluar tienen un buen desempeño.

c) La sección Escritura mostró un menor desarrollo por parte de los estudiantes que la comprensión lectora, pues nadie llegó al nivel de excelencia (nivel 5) y la gran mayoría se concentró en el nivel Suficiente (nivel 3). De todos modos, el 73,11\% de alumnos/as que alcanzan el nivel de logro (14 puntos) revela que el grupo tiene un dominio adecuado de las habilidades escriturales mínimas para su desempeño académico, es decir, son capaces de seguir una instrucción de escritura mediante la producción de un texto que se ajusta al contenido, además de tener una estructura coherente, expresada en separación de ideas en párrafos, y recursos de cohesión adecuados. 
La lectura de los puntajes 0 de las tres preguntas muestra que progresivamente hay un aumento de la deserción en la escritura, puesto que la pregunta A no fue abordada por 6 alumnos, mientras que la B por 22 alumnos; y la C, por 44.

El análisis general muestra que los estudiantes evaluados tienen un buen desempeño, con niveles de logro superiores, dada la cantidad de examinandos que aprobaron ambas secciones y la calidad de sus puntajes.

\section{Referencias bibliográficas}

CALSAMIGLIA, Helena y TUSÓN, Amparo. Las cosas del decir. Barcelona: Ariel, 2007.

CASSANY, Daniel. Describir el escribir. Cómo se aprende a escribir. Barcelona: Paidós, 2003.

COMITÉ DE LENGUAJE Y COMUNICACIÓN, DEMRE. Marco teórico prueba CodiceDerecho. Inédito, Santiago, 2011.

GARCÍA, Juan. Lectura y conocimiento. Barcelona: Paidós, 2006.

MINISTERIO DE EDUCACIÓN. Mapas de Progreso. Lectura y Producción de textos escritos. Santiago: 2008.

PÉREZ-ZORRILLA, Ma Jesús. "Evaluación de la comprensión lectora: dificultades y limitaciones". En: Revista de Educación. 2005, núm. Extraordinario. Pp. 121 - 138. Disponible en web: http://www.revistaeducacion.mec.es/re2005_08.htm.

RAMOS, Carlos. "Elaboración de un instrumento para medir comprensión lectora en niños de Octavo Año Básico”. En: Revista Onomazein. 2006, núm. 14. Pp. 197 - 210. Disponible en web: http://www.onomazein.net/Articulos/14/7_Ramos.pdf 